\title{
Projeto Helix: Explorando Social Internet of Things (SIoT) no Atendimento à Pessoas Portadoras de Deficiência Visual*
}

\author{
Felipe Haertel ${ }^{1}$, Leandro Camargo ${ }^{4}$, João Lopes $^{2}$, \\ Ana Pernas ${ }^{4}$, Jorge Barbosa ${ }^{3}$, Adenauer Yamin ${ }^{14}$ \\ ${ }^{1}$ Mestrado em Engenharia Eletrônica e Computação (MEEC) - UCPEL \\ ${ }^{2}$ Prog. de Pós-Graduação em Ciências e Tecs. na Educação (PPGCITED) - IFSul \\ ${ }^{3}$ Prog. de Pós-Graduação em Computação Aplicada (PPGCA) - UNISINOS \\ ${ }^{4}$ Prog. de Pós-Graduação em Ciência da Computação (PPGC) - UFPel \\ felipe.haertel@gmail.com, joaolopes@ifsul.edu.br, \\ jbarbosa@unisinos.br, adenauer.yamin@ucpel.edu.br \\ marilza@inf.ufpel.edu.br, leandro.camargo@inf.ufpel.edu.br
}

\begin{abstract}
This paper proposes an approach, called Helix-SIoT, which explores the Social IoT concepts to support people with visual impairments (PCDVs). The objective is to enhance the treatment of the different used sensors with the use of a SIoT. To this purpose, the proposal applies Context Awareness and Multiple Criteria Decision Analysis in the management of interactions involving Caregivers and PCDVs.
\end{abstract}

Resumo. Neste artigo é proposta uma abordagem, denominada Helix-SIoT, que explora os conceitos da Internet Social das Coisas para suporte às pessoas com deficiência visual (PCDVs). O objetivo é potencializar com o uso de uma SIoTo tratamento dos diferentes sensores envolvidos. Para tanto, a proposta emprega Ciência de Contexto e Decisão baseada em Múltiplos Critérios na gerência das interações envolvendo PCDVs e seus Cuidadores.

\section{Introdução}

$\mathrm{Na}$ Internet Social das Coisas (Social Internet of Things - SIoT), objetos conectados em rede podem fornecer serviços explorando a relação social entre humanos e objetos, humanos e humanos, e objetos e objetos. Deste modo, em uma rede social, um objeto com funcionalidades de servidor pode ser responsável por inferir situações em outros objetos, e articular relações, empregando para isto mecanismos de Ciência de Contexto [Atzori et al. 2012] [Shamszaman and Ali 2018].

O trabalho apresentado neste artigo faz parte do Projeto Helix. A abordagem cuja concepção é tratada neste artigo, denominada Helix-SIoT, tem como objetivo explorar o uso de uma SIoT no tratamento dos diferentes sensores associados tanto às pessoas com deficiência visual (PCDVs) como aos seus Cuidadores. Para tanto, emprega Ciência de Contexto e Decisão baseada em Múltiplos Critérios na gerência das interações envolvendo PCDVs e Cuidadores.

\footnotetext{
*O presente trabalho foi realizado com apoio da Coordenação de Aperfeiçoamento de Pessoal de Nível Superior - Brasil (CAPES) - Código de Financiamento 001.
} 
A Helix-SIoT está integrada ao Subsistema de Adaptação e Reconhecimento de Contexto do middleware EXEHDA [da Silva Machado et al. 2017]. O EXEHDA, enquanto middleware para IoT, provê suporte para a aquisição, armazenamento e processamento das informações de contexto necessárias as diferentes funcionalidades providas pela abordagem proposta.

O artigo está estruturado da seguinte forma: a Seção 2 apresenta a revisão de literatura realizada. A abordagem Helix-SIoT e suas funcionalidades são apresentadas na seção 3. A Seção 4 destaca os cenários de uso. Por fim, na Seção 5 são feitas as considerações finais.

\section{Revisão de Literatura}

Diversos trabalhos foram identificados quando da revisão de literatura. Nesta Seção estão contemplados aqueles cujo tema de pesquisa mais se aproxima da abordagem proposta neste artigo, a qual tem por foco o emprego de uma SIoT para auxiliar na interação entre usuários portadores de objetos inteligentes, operando com as tecnologias usuais da Internet das Coisas.

[Kowshalya and Valarmathi 2017] propõem um esquema de Gerenciamento de Confiança para a SIoT, onde a confiança entre objetos é calculada com base em métricas oriundas da literatura. Desta forma, todos objetos dispostos a colaborar precisam calcular a confiança entre seus pares na perspectiva de criar uma SIoT confiável no que diz respeito ao sucesso do avanço das interações.

[Lin and Dong 2017] propõem um modelo de confiança na SIoT, no qual a confiança é um processo que não se resume a uma avaliação em relação a outro agente da SIoT, mas considera aspectos comportamentais na tomada de decisão. Assim, os agentes administradores confiam nos credores sobre seu comportamento considerando um contexto específico. Se o contexto for alterado, a decisão do agente administrador pode ser diferente. $\mathrm{O}$ contexto é baseado em dois componentes, o tipo de tarefa e o ambiente.

[Wang et al. 2016] apresentam um modelo de confiabilidade para a SIoT com o objetivo de descobrir serviços e recursos, onde a nuvem social fornece funções de computação e armazenamento e funciona como um provedor de serviços para conectar objetos de usuários finais com entidades sensoriais. Entidades sensoriais recebem tarefas e recompensas de um provedor de serviços e os realimentam com dados. A troca de mensagens entre os objetos participantes da SIoT explora a troca de links para os dados sociais considerados.

[Nitti et al. 2013] contemplam o problema de como as informações fornecidas pelos membros de uma SIoT devem ser processadas para criar um sistema confiável. As informações são produzidas a partir de padrões comportamentais dos objetos. São definidos dois modelos de gerenciamento de confiabilidade: (i) no modelo subjetivo, cada nó calcula a confiabilidade de seus amigos com base em sua própria experiência e na opinião dos amigos em comum com os provedores de serviços em potencial; (ii) no modelo objetivo, as informações sobre cada nó são distribuídas e armazenadas usando uma estrutura da Tabela Hash Distribuída, para que qualquer nó possa fazer uso das informações.

A discussão destes diferentes trabalhos relacionados foi essencial para que a concepção da Helix-SIoT tivesse bases sólidas para ser desenvolvida. Embora as pro- 
postas de exploração de SIoT nestes trabalhos não sejam especificamente direcionadas a PCDVs, os critérios de confiabilidade considerados, contemplam as demandas previstas para seleção de cuidadores, a qual tem por base escolher o Cuidador mais confiável para atuar em um determinado momento.

\section{Helix-SIoT: Abordagem Proposta}

A questão central perseguida na Helix-SIoT é o atendimento das necessidades dos envolvidos tendo como pressupostos de concepção: potencializar a autonomia, minimizar esforços de configuração e promover uma operação o mais transparente possível para as PCDVs. Para tanto, a abordagem explora Ciência de Contexto na Internet das Coisas com recursos da computação móvel, constituindo uma SIoT, onde os objetos inteligentes da IoT podem interoperar buscando uma melhor sinergia. Considerando estes aspectos foram concebidas as funcionalidades da Helix-SIoT, cuja visão geral é exibida na Figura 1.

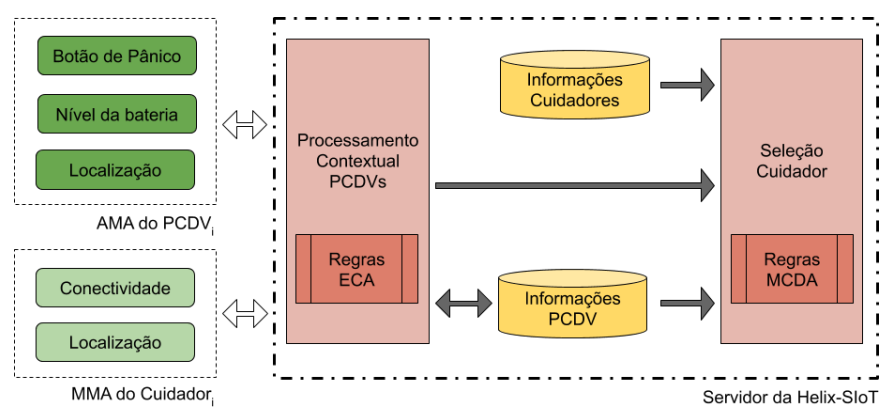

Figura 1. Visão Geral da Helix-SloT

As PCDVs e seus Cuidadores devem utilizar smartphones com recurso de GPS (Global Positioning System) nos quais os aplicativos móveis da Helix-SIoT são instalados. As diferentes parametrizações para operação da Helix-SIoT ficam a cargo de um dos cuidadores, denominado Cuidador Principal. Este cuidador escolhido pela PCDV junto com sua família, tem possibilidade de configurar os parâmetros que definem os comportamentos dos diferentes serviços da arquitetura. Outros dois tipos de Cuidadores previstos são o Corporativo, provido por uma empresa parceira, e o Cuidador do Helix, que se apresenta como uma alternativa quando todos outros cuidadores de uma PCDV não responderem.

O Assistente Móvel de Acessibilidade (AMA) é o aplicativo instalado nos smartphones que ficam de posse das PCDVs. Ele é responsável por prestar suporte diretamente na obtenção de informações contextuais. O AMA também dispõe de um Botão de Pânico, o qual uma vez acionado envia ao servidor da SIoT uma solicitação de ajuda associada a posição atual das PCDVs. Como a localização dos PCDVs é informada periodicamente pelo AMA ao servidor da Helix-SIoT, é possível gerar alertas de segurança toda vez que as PCDVs se afastarem além da distância especificada, considerando os pontos de referência previamente cadastrados.

O Monitor Móvel de Acessibilidade (MMA) interage através de um Bot configurado para o aplicativo Telegram instalado no smartphone dos Cuidadores das PCDVs (vide Figura 2). O objetivo do MMA é informar aos Cuidadores responsáveis situações das PCDVs por meio de notificações enviadas pelo Servidor da Helix-SIoT. Quando 
ocorre a notificação de uma emergência pelas PCDVs, os usuários do MMA recebem junto com a solicitação de ajuda, a posição via GPS das PCDVs, na forma de um link para o Google Maps.

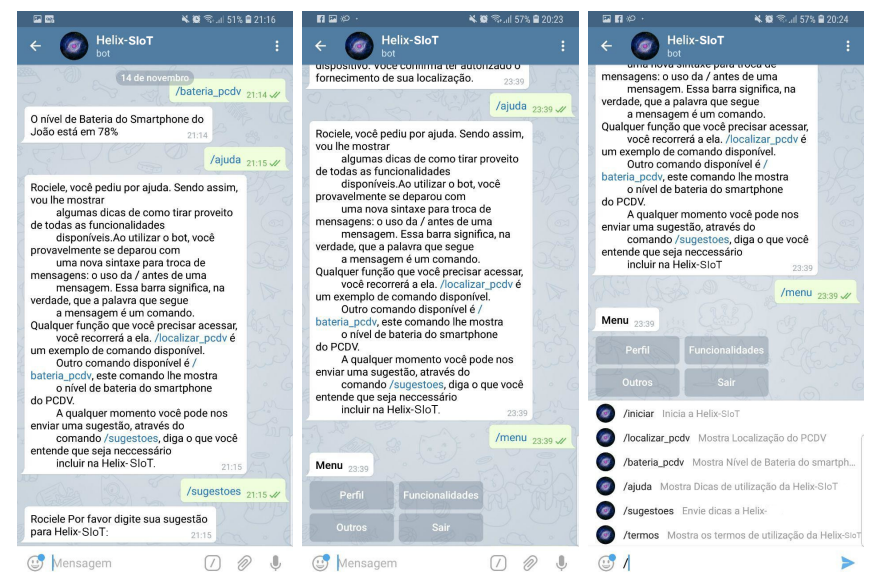

Figura 2. Notificações Enviadas aos Cuidadores das PCDVs

O Servidor da Helix-SIoT utiliza a infraestrutura de software do Servidor de Contexto do middleware EXEHDA, particularmente o Módulo de Processamento [da Silva Machado et al. 2017]. As funcionalidades de SIoT do Helix são providas por regras do tipo ECA (Evento-Condição-Ação), combinadas com a Análise de Decisão de Múltiplos Critérios (MCDA - Multiple Criteria Decision Analysis) [Marttunen et al. 2017].

Nessa perspectiva, a Helix-SIoT ganha a capacidade de avaliar o impacto de um conjunto ponderado de alternativas. Este peso atribuído aos diferentes critérios permite a criação de uma escala, denominada de "escala de utilidade" [Whaiduzzaman et al. 2014].

As Informações Contextuais, relacionadas a seguir, são coletadas tanto pelos AMAs dos PCDVs, como pelos MMAs dos Cuidadores, e disponibilizadas para tomada de decisão pela Helix-SIoT.

- Beacon de Atividade: para manter a Helix-SIoT funcional, tanto a PCDV quanto os Cuidadores devem enviar informações periodicamente ao longo do dia, sendo o intervalo de tempo pré-configurado pelo Cuidador Principal, essas informações denominadas de Beacon de Atividade, determinam se a PCDV e seus respectivos Cuidadores estão ativos na rede. Caso algum Cuidador não envie o Beacon de Atividade, ele perde pontos na Seleção de Cuidadores da Helix-SIoT. No caso da PCDV, se o Beacon de Atividade não for enviado, é gerado um alerta à Rede de Cuidadores, com a última localização disponível da PCDV no Servidor.

- Nível de Bateria: a cada Beacon de Atividade, é enviado o nível de bateria dos smartphones, tanto das PCVDs, quanto dos Cuidadores. No caso das PCDVs, é utilizado para eventuais disparos de solicitações de atendimento para a Rede de Cuidadores, enquanto para os Cuidadores, é utilizado como critério para evitar o envio de notificações a Cuidadores que estejam com um nível crítico de bateria e com isso possam ter seu atendimento a PCDV comprometido após aceitar a tarefa.

- Localização: os smartphones da PCDV e de seus Cuidadores disponibilizam as suas localizações ao servidor da Helix-SIoT. O servidor considera essas 
informações na tomada de decisão multicritério, priorizando assim notificar o Cuidador que tiver menor trajetória a percorrer para chegar até a PCDV.

Além destas três informações, é considerado como informação contextual da PCDV o acionamento do Botão de Pânico, que envia uma mensagem com a localização atual da PCDV ao servidor da Helix-SIoT. A Helix-SIoT por padrão notifica o Cuidador mais próximo a PCDV, conforme as últimas localizações enviadas. Este padrão de notificação pode ser alterado pelo Cuidador Principal, entre as seguintes opções: Preferência de Turno, Cuidador Responsável e Distância.

Os Cuidadores podem escolher os turnos de preferência para receber as notificações, sendo considerados para cada dia da semana: manhã, tarde, noite e madrugada. Esta preferência é dividida em quatro níveis: indisponível, baixo, médio e alto.

Com base nas informações contextuais coletadas das PCDVs, as situações, apresentadas a seguir, podem ser identificadas empregando regras do tipo ECA. As regras ECA tratam eventos gerados a partir das seguintes mudanças no estado dos contextos:

- Nível Baixo de Bateria: o Cuidador Principal configura os níveis de bateria a serem considerados e a prioridade de atendimento para cada um.

- Afastamento das Regiões de Vivência Previstas: são informados na Aplicação de Gerenciamento da Helix-SIoT, pelo Cuidador Principal, os locais de vivência habitual da PCDV, através de coordenadas disponibilizadas pelo Google Maps [Brown et al. 2018]. Junto a cada coordenada, deve ser informada uma distância de perímetro permitida, garantindo que a PCDV possa se deslocar sem que isso gere notificações desnecessárias.

- Taxa de Descarga da Bateria: a cada Beacon de Atividade informado, é passado o nível de bateria atual do celular. Por padrão se houver um nível de descarga superior a $20 \%$ é gerado um alerta a Rede de Cuidadores. O Cuidador Principal pode alterar o nível de descarga a ser considerado para geração de alertas.

O envio de notificações aos Cuidadores utiliza o algoritmo MCDA Simple Additive Weight (SAW) para auxiliar no julgamento da tomada de decisão, definindo qual Cuidador deve receber as notificações, sendo adotado um peso de 0 a 1, com uma casa decimal, para cada um destes aspectos: (i) Distância entre PCDV e Cuidador em metros; (ii) Disponibilidade: a disponibilidade será calculada levando em consideração a preferência de turnos especificada no cadastro dos Cuidadores; (iii) Comunicabilidade: será realizado o cálculo do potencial de comunicabilidade sempre que o nível de bateria do smartphone do Cuidador ficar abaixo de um nível pré-definido de carga, pelo Cuidador Principal; e (iv) Confiança: Será calculada com base na disponibilidade do Cuidador para o turno de atendimento. Também será empregada uma amortização calculada com base no histórico de atendimento das solicitações realizadas pela PCDV.

A normalização para a Distância entre PCDV e Cuidador é realizada através do algoritmo SAW, utilizando a Equação 1, onde $\min \left(d t_{j}\right)$ representa o menor valor entre todos os Cuidadores da Rede e $d t_{i, j}$ o Cuidador que será calculado [Tzeng and Huang 2011].

$$
D t_{i, j}=\left[\frac{\min \left(d t_{j}\right) * 100}{d t_{i, j}}\right]
$$


O resultado final do MCDA é calculado considerando o peso estabelecido pelo Cuidador Principal para cada um destes aspectos, conforme Eq. 2, onde $D t_{i, j}$ se refere ao valor normalizado da Distância do Cuidador, $P d t$, ao peso referente a Distância, $D p_{i, j}$ a Disponibilidade, sendo $P d p$ o seu peso e $C b_{i, j}$ representa a Comunicabilidade, assim como $P c b$ o seu peso, a Confiança é representada como $C f_{i, j}$ e seu peso por $P c f$.

$$
M C D A=D t_{i, j} * P d t+D p_{i, j} * P d p+C b_{i, j} * P c b+C f_{i, j} * P c f
$$

\section{Helix-SIoT: Cenários de Uso}

A avaliação da Helix-SIoT foi baseada em dois Cenários de Uso, os quais contemplam uma visão geral das principais funcionalidades da abordagem proposta.

Considerando a necessidade de ter diferentes situações a serem tratadas, tanto na perspectiva das PCDVs, como dos Cuidadores, a abordagem utilizada considerou realizar uma produção sintética de valores para os sensores, respeitando a faixa operacional usual disponível na literatura, bem como a opinião da comunidade da Associação Escola Louis Braille $^{1}$, a qual é detentora de significativa experiência no trato de PCDVs.

\subsection{Cenário 1 - Processamento Contextual dos Sensores das PCDVs}

Este cenário explora as funcionalidades do processamento contextual dos dados produzidos pelos sensores associados às PCDVs. A partir destes dados, as diferentes funcionalidades da Helix-SIoT foram trabalhadas individualmente, bem como foi realizada a interoperação dos diferentes componentes arquiteturais.

Os dados empregados, produzidos sinteticamente, referentes ao sensoriamento das PCDVs, foram entregues utilizando a API padrão da arquitetura da Helix-SIoT, emulando o comportamento de sensores reais. Neste cenário são considerados os sensores de Nível de Bateria, Localização e Beacon de Atividade.

Algumas informações foram parametrizadas pelo Cuidador Principal, para a realização das emulações: (i) níveis de bateria menores que $20 \%$ e maiores que $15 \%$, com prioridade baixa, menores que $15 \%$ e maiores que $5 \%$ com prioridade média e menores que $5 \%$ com prioridade alta; (ii) Beacon de Atividade enviado a cada 30 minutos, com tolerância de 10 minutos.

Neste cenário, foram gerados 1008 Beacons de Atividades com informações sobre os sensores considerados, sendo simulado o envio de um Beacon a cada 30 minutos, durante três semanas. A partir das informações contextuais entregues pelos Beacons a Helix-SIoT, referentes aos sensores das PCDVs, foram identificadas situações, cujos totais podem ser vistos na Tabela 1.

\subsection{Cenário 2 - Seleção Multicritério de Cuidadores}

Este cenário explora a utilização do algoritmo SAW na tomada de decisão baseada em múltiplos critérios para a seleção de Cuidadores. O Cuidador Principal parametriza algumas informações para a realização das emulações, considerando que a PCDV possui quatro Cuidadores ativos cadastrados e que os pesos de cada um dos aspectos foram configurados da seguinte forma: (i) Distância em 0.6; (ii) Disponibilidade em 0.5; (iii) Comunicabilidade em 0.8; e (iv) Confiança em 0.7.

\footnotetext{
${ }^{1}$ https://louisbraille.org.br/
} 
Tabela 1. Situações Identificadas pela Helix-SloT

\begin{tabular}{l|c}
\hline Situações & Totais \\
\hline Taxa de Descarga da Bateria superior a 20\% entre Beacons & 35 \\
Nível baixo de Bateria - Prioridade Baixa & 27 \\
Nível baixo de Bateria - Prioridade Média & 21 \\
Nível baixo de Bateria - Prioridade Alta & 4 \\
Afastamento das Regiões de Vivência Previstas & 27 \\
Beacon não enviado por falta de comunicação & 36 \\
Nenhuma Situação Identificada & 858 \\
\hline
\end{tabular}

Também, foi definida pelo Cuidador Principal a disponibilidade de cada um dos quatro cuidadores. A distância de cada Cuidador foi calculada com base em sua localização comparada a localização da PCDV, considerando o deslocamento da PCDV e dos seus Cuidadores. Neste Cenário foi considerado que o Cuidador 1, fica em distâncias entre 1 metro e $2 \mathrm{Km}$, o Cuidador 2 e 4 em distâncias entre 1 metro e $5 \mathrm{Km}$ e o Cuidador 3 nos turnos da manhã e tarde fica em distância entre $3 \mathrm{Km}$ a $5 \mathrm{Km}$ e a noite e madrugada entre 1 metro e $5 \mathrm{Km}$.

Foi considerado um percentual de comunicabilidade acima de $80 \%$ dos casos, fazendo que os Cuidadores sem comunicabilidade percam pontos e dessa forma não sejam priorizados para o atendimento. Com base nisto, foi considerado o índice de confiança do Cuidador, o qual é utilizado levando em consideração a sua preferência de atendimento para aquele turno. Dessa forma, cada cuidador terá três níveis de confiança: Alta, Média e Baixa. Este índice varia conforme as solicitações e atendimentos do Cuidador.

Para este cenário, foram realizados testes de solicitação de atendimento em todos os turnos cadastrados, para os 7 dias da semana, totalizando 28 turnos por semana, rodando por 36 semanas, resultando 1008 testes. Nos testes realizados, em mais de 92\% das solicitações, o atendimento foi realizado por um dos quatro Cuidadores cadastrados, sendo 1123 solicitações e 933 atendimentos confirmados, totalizando aproximadamente $83 \%$, conforme mostra a Figura 3.

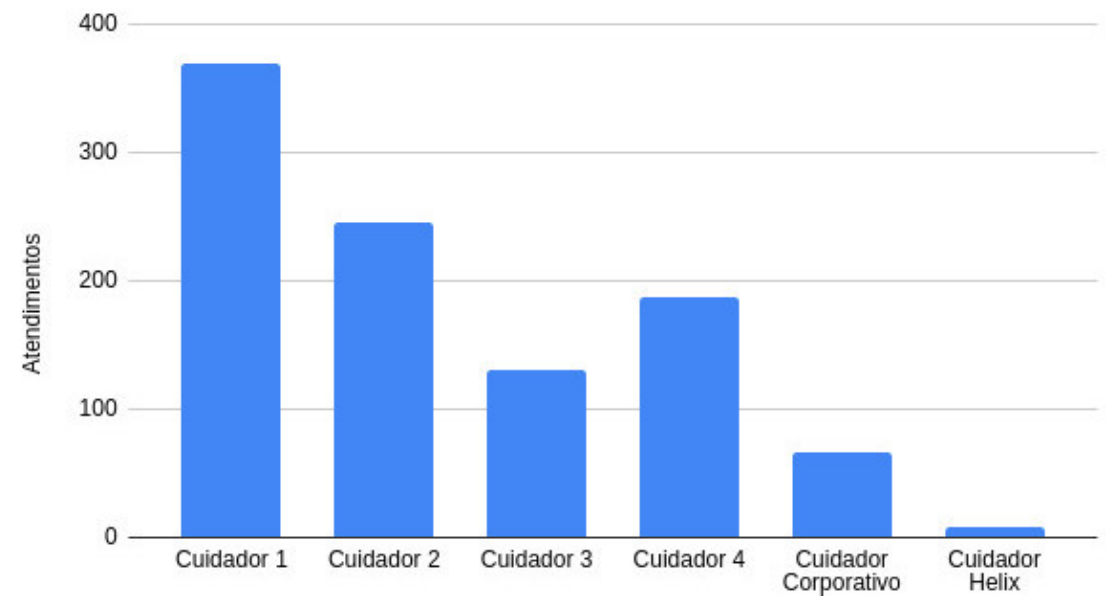

Figura 3. Atendimentos dos Cuidadores 


\section{Considerações Finais}

Os resultados obtidos apontam que o emprego de uma SIoT que explore Ciência de Contexto para identificação de situações das PCDVs, e uma abordagem baseada em MCDA para seleção dos Cuidadores, como discutido nos Cenários de Uso, pode potencializar a cooperação entre usuários de dispositivos que sejam dotados de recursos de sensoriamento e interconectados pela IoT.

Como trabalhos futuros, destacam-se as seguintes frentes de atividades: (i) submeter um estudo de campo da abordagem Helix-SIoT a um Comitê de Ética a fim de realizar testes com PCDVs; (ii) revisar os aspectos de segurança da infraestrutura hardware/software empregada na Helix-SIoT; e (iii) explorar o uso dos históricos de dados para registro dos deslocamentos e também análises de dados.

\section{Referências}

Atzori, L., Iera, A., Morabito, G., and Nitti, M. (2012). The social internet of things (siot)-when social networks meet the internet of things: Concept, architecture and network characterization. Computer Networks, 56(16):3594-3608.

Brown, J., Zhou, Z., and Chow, Y.-W. (2018). Metamorphic testing of navigation software: A pilot study with google maps. Hawaii Int. Conference on System Sciences.

da Silva Machado, R., Almeida, R. B., da Rosa, D. Y. L., Lopes, J. L. B., Pernas, A. M., and Yamin, A. C. (2017). EXEHDA-HM: A compositional approach to explore contextual information on hybrid models. Future Gener. Comput. Syst., 73:1-12.

Kowshalya, A. M. and Valarmathi, M. (2017). Trust management in the social internet of things. Wireless Personal Communications, 96(2):2681-2691.

Lin, Z. and Dong, L. (2017). Clarifying trust in social internet of things. IEEE Transactions on Knowledge and Data Engineering, 30(2):234-248.

Marttunen, M., Lienert, J., and Belton, V. (2017). Structuring problems for multi-criteria decision analysis in practice: A literature review of method combinations. European Journal of Operational Research, 263(1):1-17.

Nitti, M., Girau, R., and Atzori, L. (2013). Trustworthiness management in the social internet of things. IEEE Trans. on knowledge and data engineering, 26(5):1253-1266.

Shamszaman, Z. U. and Ali, M. I. (2018). Toward a smart society through semantic virtual-object enabled real-time management framework in the social internet of things. IEEE Internet of Things Journal, 5(4):2572-2579.

Tzeng, G. H. and Huang, J. J. (2011). Multiple Attribute Decision Making: Methods and Applications. A Chapman \& Hall book. Taylor \& Francis, New York.

Wang, K., Qi, X., Shu, L., Deng, D.-j., and Rodrigues, J. J. (2016). Toward trustworthy crowdsourcing in the social internet of things. IEEE Wireless Comms., 23(5):30-36.

Whaiduzzaman, M., Gani, A., Anuar, N. B., Shiraz, M., Haque, M. N., and Haque, I. T. (2014). Cloud service selection using multicriteria decision analysis. The Scientific World Journal, 2014:10. 